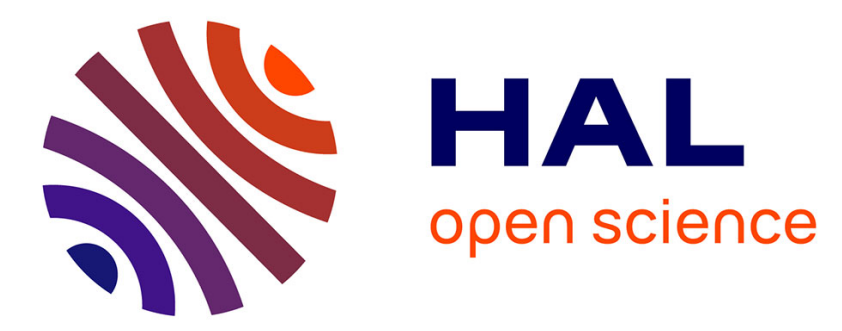

\title{
La question régionale dans l'espace roumain
}

Violette Rey

\section{- To cite this version:}

Violette Rey. La question régionale dans l'espace roumain. Espace Géographique, 1994, 23 (4), pp.361-376. 10.3406/spgeo.1994.3337 . hal-02886811

\section{HAL Id: hal-02886811 https://hal.science/hal-02886811}

Submitted on 1 Jul 2020

HAL is a multi-disciplinary open access archive for the deposit and dissemination of scientific research documents, whether they are published or not. The documents may come from teaching and research institutions in France or abroad, or from public or private research centers.
L'archive ouverte pluridisciplinaire HAL, est destinée au dépôt et à la diffusion de documents scientifiques de niveau recherche, publiés ou non, émanant des établissements d'enseignement et de recherche français ou étrangers, des laboratoires publics ou privés. 


\section{La question régionale dans l'espace roumain}

\section{Violette Rey}

\section{Citer ce document / Cite this document :}

Rey Violette. La question régionale dans l'espace roumain. In: Espace géographique, tome 23, n4, 1994. pp. 361-376; doi : https://doi.org/10.3406/spgeo.1994.3337

https://www.persee.fr/doc/spgeo_0046-2497_1994_num_23_4_3337

Fichier pdf généré le 02/04/2018 
Problema regionalâ în spajiul românesc. - Sfârsjt al colectivismului §̧i al unei dictaturi personale, început al unei «tranzijii» : Romania înfruntâ dubla sfidare a unei re-aproprieri a teritorialitâtii cotidiene §i a coeziunii dintre subsistemele régionale care compun teritoriul national. Analizele multivariate asupra datelor jude^ene din 1969, 1989 §i 1993 aratâ câ transfor- mârile nu sunt aleatoare, ci ascultâ de logica spatialâ a evolujiei îndelungate, respectiv aceea a mediului §̧i a istoriei, înscrisâ în tipurile de comportament demografic. Indelungata emergenjâ a unei structuri centrate pe o axa transversalâ NVSE a fost inhibatâ prin difuzia unei omogeneitâçi paralizante, urmata de dezgroparea vechilor structuri integrate §i de avântul luat de anumite regiuni periferice. Aceastâ panoplie haoticâ de configura^ este expresia concretâ a proceselor spatiale spécifiée unei arii d'entre-deux care fac deosebit de complexe construc ${ }^{\wedge}$ iile identitare.

\begin{abstract}
Space and the regional problem in Romania. - Now that socialism and personal dictatorship have ended, 'transition' has started. Romania is at present confronted with a twofold challenge: reappropriating territory on the basis of everyday life and reestablishing cohesion between the subregions which make up its national territory. Multivariate analyses at departmental level based on data for 1969, 1989 and 1993 show that changes, far from being a random phenomenon, are the result of the long-term spatial logic of environment and history as operated by different types of demographic behaviour. The country was gradually becoming centralised around a NW/SE axis when this process was stopped by the paralysing effects of the attempt at diffusing homogeneity, followed by the artificial revival of former nested structures and the growing importance of emerging peripheral regions. This chaotic series of configurations is the concrete illustration of the specific spatial processes typical of a transition era in which the construction of identity is rendered particularly difficult.
\end{abstract}

\title{
Résumé
}

Fin du socialisme, fin d'une dictature personnelle, début d'une «transition»: la Roumanie est confrontée au double défi d'une réappropriation de la territorialité au quotidien et de la cohésion entre les sousensembles régionaux du territoire national. La dynamique des structures socio-économiques départementales est étudiée diachroniquement avec des méthodes d'analyse multivariée (1969, 1989, 1993). L'ampleur de la mutation structurelle en 20 ans, vers une uniformité industrielle et urbaine, est suivie d'une réponse géographique non aléatoire au choc de 1989, relevant d'une logique spatiale des héritages de très longue durée, et assortie de types différents de comportements démographiques. La Roumanie a ainsi connu successivement l'émergence d'une centralité selon un axe transversal NOSE, la diffusion d'une homogénéisation paralysante, l'exhumation de structures emboîtées traditionnelles et un avantage nouveau à certaines régions périphériques. Ces processus spatiaux caractéristiques d'une aire d'entre-deux compliquent les constructions identitaires. 


\title{
La question régionale dans l'espace roumain
}

\author{
Violette Rey \\ École Normale Supérieure de Fontenay - Saint-Cloud, Équipe P.A.R.I.S.
}

RÉSUMÉ-Fin du socialisme, fin d'une dictature personnelle, début d'une «transition»: la Roumanie est confrontée au double défi d'une réappropriation de la territorialité au quotidien et de la cohésion entre les sous-ensembles régionaux du territoire national. La dynamique des structures socio-économiques départementales est étudiée diachroniquement avec des méthodes d'analyse multivariée $(1969,1989,1993)$. L'ampleur de la mutation structurelle en 20 ans, vers une uniformité industrielle et urbaine, est suivie d'une réponse géographique non aléatoire au choc de 1989. relevant d'une logique spatiale des héritages de très longue durée, et assortie de types différents de comportements démographiques. La Roumanie a ainsi connu successivement l'émergence d'une ccntralitć sclon un axc transversal NO-SE, la diffusion d'une homogénéisation paralysante, l'exhumation de structures embồtées traditionnelles et un avantage nouveau à certaines régions périphériques. Ces processus spatiaux caractéristiques d'une aire d'entre-deux compliquent les constructions identitaires.

ESPACE TAMPON, INTÉGRATION RÉGIONALE, MÉTHODES QUANTITATIVES, ROUMANIE, SYSTĖME NATIONAL., TRANSITION POSTSOCIALISTE

REzuMAT.- Problema regională în spaţiul românesc. - Sfârşit al colectivismului şi al unei dictaturi personale. început al unei «tranziţii» : România înfruntă dubla sfidare a unei re-aproprieri a teritorialităţii cotidiene şi a coeziunii dintre subsistemele regionale care compun teritoriul naţional. Analizele multivariate asupra datelor judeţene din 1969, 1989 şi 1993 arată că transformările nu sunt aleatoare, ci ascultă de logica spatială a evoluţiei îndelungate, respectiv aceea a mediului şi a istoriei, înscrisă în tipurile de comportament demografic. Îndelungata emergenţă a unei structuri centrate pe o axă transversală NV-SE a fost inhibată prin difuzia unci omogeneităţi paralizante, urmată de dezgroparea vechilor structuri integrate şi de avântul luat de anumite regiuni periferice. Această panoplie haotică de configuraţii este expresia concretă a proceselor spaţiale specifice unei arii d'entre-deux care fac deosebit de complexe construcţiile identitare.

INTEGRARE REGIONALĂ, METODE CANTITATIVE, ROMÂNIA, SISŢEM NATIONAL, SPAŢIU D'ENTRE-DELX, TRANZIŢIE. POST-SOCIALISTĂ

ABSTRACT. - Space and the regional problem in Romania.Now that socialism and personal dictatorship have ended, 'transition' has slarted. Rumania is at present confronted with a twofold challenge: reappropriating territory on the basis of everyday life and reestablishing cohesion between the sub-regions which make up its national territory. Multivariate analyses at departmental level based on data for 1969, 1989 and 1993 show that changes, far from being a random phenomenon, are the result of the long-term spatial logic of environment and history as operated by different types of demographic behaviour. The country was gradually becoming centralised around a NW/SE axis when this process was stopped by the paralysing effects of the attempt at diffusing homogeneity, followed by the artificial revival of former nested structures and the growing importance of emerging peripheral regions. This chaotic series of configurations is the concrete illustration of the specific spatial processes typical of a transition era in which the construction of identity is rendered particularly difficult.

NATIONAL, SYSTEM, POST-SOCIALIST TRANSITION, QLANTITATIVE. METHODS, REGIONAL INTEGRATION, ROMANIA, SPACE IN-BETWEEN 
La fin du $\mathrm{XX}^{\mathrm{c}}$ siècle oblige à reconsidérer les fondements de l'analyse régionale en Roumanie. La mondialisation de l'économie guide partout les filières et les spécialisations; la multiplicité des réseaux d'appartenance sociale, jouant à des échelles très différentes, des réseaux professionnels à ceux de la vie privée, paraissent défier un cadre commun d'intégration identitaire qui serait l'échelon régional. D'autres phénomènes montrent au contraire la vitalité de ce niveau intermédiaire d'organisation, qu'il s'agisse des «régions qui gagnent» et des «régions qui perdent» dont les dynamiques divergentes alimentent les débats sur l'aménagement et la gestion des territoires, ou qu'il s'agisse de la montée en puissance de certaines régions, remettant en question leur niveau supérieur d'appartenance étatique.

L'expression «les régions contre l'État» n'est pas une vaine périphrase dans l'Europe de la fin du $\mathrm{Xx}^{\mathrm{e}}$ siècle, qui voit progresser les entités territoriales intermédiaires. Le passage à des constitutions fédéralistes en Europe occidentale et l'éclatement de certains États d'Europe centre-orientale en unités plus petites, à dimension régionale mais ramassées autour d'une très forte cohésion identitaire de nationalité, en sont l'expression. Ces processus peuvent correspondre à un ajustement aux changements technologiques et organisationnels de la sphère économique, au niveau mondial pour les entreprises et au niveau régional pour les sociétés; ils sont tout autant l'expression d'autres manières de vivre et d'adhérer aux valeurs identifiées à des ensembles culturels. La problématique régionale apparaît donc comme un défi toujours renouvelé de l'analyse des espaces humanisés. La relance des approches théoriques à son sujet s'inscrit dans ce paradigme plus général de la différence et de la diversité, redevenues valeurs cardinales, et du statut scientifique à leur définir. Nous traitons concrètement de cette question à travers le cas de l'espace roumain, revisité 25 ans après.

\section{Les défis d'une problématique régionale}

\section{Les significations d'arrière-plan}

L'observateur étranger souligne souvent le caractère national et même nationaliste des comportements roumains, associés à la montée générale des revendications identitaires surgies des décombres du système socialiste en Europe centre-orientale. Dans les conversations et les débats internes. il est frappant de constater combien actuellement les Roumains aiment à se situer en fonction de leur origine régionale et de l'appartenance historique de leur province. Cette double attitude s'alimente probablement à la source même du rejet du système précédent qui, pour exister en tant que tel, avait trop largement fait fi du besoin d'identité propre à chaque groupe social. L'homme socialiste nouveau, à la fois gorgé du principe internationaliste et du patriotisme du «peuple roumain», était perçu comme une fiction surimposée à la réalité d'un vécu faite de multiples aliénations quotidiennes et symboliques.

Privé des apports que les échanges et les ouvertures donnent à la construction de l'identité individuelle et collective, le besoin d'identité s'était paradoxalement réfugié et concentré sur sa composante territoriale (1), dont les références régionalistes actuelles sont la manifestation. À cela s'ajoutent les caractéristiques mêmes de la transition postsocialiste. L'effort de suppression du système de commandes totalement centralisé s'accompagne d'un réel effondrement de l'échelon national comme cadre d'organisation, d'une dévalorisation du modèle étatique et d'un repli sur le local, cadre concret d'un quotidien incertain et au sein duquel on tente de surmonter l'accumulation des difficultés pratiques de tous ordres. L'expression politisée la plus manifeste de l'attitude régionaliste apparaît dans la promotion d'un retour à l'ordre territorial présocialiste, tel qu'il fonctionnait dans le cadre de la départementalisation administrative d'avant-guerre, et tel que le réclame un petit parti politique dans l'obédience de l'opposition gouvernementale, particulièrement animé par les «départements supprimés» (2).

Au-delà des enjeux de la territorialité au quotidien, existe un enjeu régional, qui pose la question du degré de cohésion de l'État. Or la Roumanie est une entité récente, née en

(1) V. Rey in Radvanyi J., Rfy V. (1987). Régions et pouvoirs régionaux en Europe de l'Est et en URSS. Paris: Masson, p. 16.

(2) IoRDAN I., Rey V. (1994). «La carte administrative de la Roumanie: probable ou impossible retour à l'état d'avant-guerre?» Bulletin de la Société languedocienne de géographie, $\mathrm{n}^{\circ} 4$. 
1918 des traités de la fin de la première guerre mondiale, qui ont rassemblé la Roumanie dite du Vieux Royaume (elle-même née en 1859 de l'union des deux principautés de Moldavie et de Valachie) avec la Transylvanie et le Banat, anciennes provinces de l'empire d'Autriche-Hongrie. Par cet acte, disparaissait politiquement un tronçon de la limite multiséculaire entre l'Europe centrale d'influence germanique et l'Europe sud-orientale d'influence russe et ottomane. L'association de ces territoires aux passés si différents, afin de construire un ensemble politique intégré, fut le défi initial du nouvel État roumain. Ce défi, décrit par $\mathrm{E}$. de Martonne dans l'entre-deux-guerres (3), était d'autant plus difficile que la présence de fortes minorités allemandes et hongroises en Transylvanie alimentait des courants irrédentistes, qui facilitèrent l'attribution de la Transylvanie du Nord à la Hongrie entre 1940 et 1945.

Dans son enveloppe actuelle, issue de la seconde guerre mondiale, la Roumanie n'a plus la Bucovine du Nord (qui est en Ukraine), la Bessarabie (devenue République moldave d'URSS puis Moldavie indépendante) et le sud de la Dobroudja. Les estimations des derniers recensements donnent la proportion de population de langue roumaine à $19 \%, 65 \%$ et $0 \%$. Les deux ingrédients qui font de l'Europe centre-orientale un espace fragile existent donc en Roumanie: le territoire est historiquement composite, sillonné par une ancienne fracture politique partiellement doublée d'une limite religieuse (entre le christianisme orthodoxe et les christianismes occidentaux); la présence de minorités aiguise le souci des affirmations identitaires et en exacerbe facilement les passions.

Tel est donc l'arrière-plan complexe qui, en ce moment, donne son sens à toute analyse des structures spatiales et des processus de régionalisation en Roumanie: d'une part

(3) Géographie Universelle, vol. «Europe Centrale», 1930, Paris: A. Colin. Il soulignait combien à ces deux passés étaient associées une très sensible disparité des niveaux de développement économique et matériel, plus avancé à l'Ouest, plus arriéré à l'Est, de même que des expressions très différentes de la vie culturelle et de la vie sociopolitique. Le cas n'était d'ailleurs pas propre à la Roumanic; Pologne. Tchécoslovaquie. Yougoslavie étaient confrontées à de semblables problèmes liés à des couplages de territoires aux passés disjoints. les enjeux de la territorialité au quotidien, qui éclairent les formes de recomposition locale et régionale; de l'autre, la poursuite de la construction du territoire étatique proprement dit.

\section{Le concept de région}

L'analyse des structures spatiales de la Roumanie contemporaine se fonde ici sur l'idée de région comme combinaison dynamique entre des phénomènes d'identité et des mécanismes fonctionnels. Cette combinaison est porteuse d'une énergie spécifique qui déforme, organise, polarise l'étendue géographique (4). À la dimension identitaire se rattache plutôt la capacité d'une autoreproduction, ce que l'on peut assimiler à une force de la longue durée. L'espace régional est la matérialisation d'une mémoire qui fournit des supports aux modes de représentations symboliques et aux systèmes de valeurs des groupes qui l'habitent et qui agissent (en partie) dans le sens de la conservation et de l'affirmation de cette identité. À la dimension fonctionnelle se rattache plutôt la force de l'efficacité du temps présent, la capacité d'adaptation et de transformation (qui peut même conduire jusqu'aux transferts hors d'une région si la fonctionnalité qu'offre la région disparait).

L'identification régionale et sa longévité sont probablement d'autant plus marquées que l'interaction entre les deux dimensions est plus forte, en particulier que les traits de fonctionnalité acquièrent eux-mêmes une longue durée, comme le montre le rôle du réseau urbain et de la fonctionnalité des lieux centraux dans la construction d'un espace régional. De multiples articulations entre ces deux dimensions identitaire et fonctionnelle donnent un éventail de types régionaux, allant de la consolidation réciproque à l'antagonisme. Les travaux sur les lieux de dynamique innovante soulignent comment l'innovation s'insère précisément à la charnière des deux dimensions, et insistent sur le facteur dit de l'«autopoèse», lié principalement à l'identité (G. Dematteis).

(4) Plusieurs articles récents ont dressé l'état des conceptualisations du fait régional et de son approche, voir la bibliographic. 
La proximité géographique sert d'hypothèse centrale pour comprendre ce qui favorise les synergies multiples, et facilite les combinaisons dynamiques entre identité et fonction, porteuses du processus régional. Ces combinaisons construisent des homogénéités complexes (5), qui donnent précisément la lisibilité et l'individualisation extérieure de la région dans un ensemble géographique plus vaste. Sont alors associées à l'identification des régions l'idée de leur centre et l'idée de leurs limites. Par contre, la région n'a pas d'indépendance de décision; elle est un sous-ensemble d'un système de pouvoir territorial plus large, même quand la structure fédérale lui donne une réelle autonomie; d'où le caractère à la fois très durable de la conscience régionale, qui n'est pas impulsée d'en haut ou d'ailleurs, et la difficulté à en saisir les contours réels.

\section{La dynamique des structures départementales, 1969-1989}

L'approche d'une réalité aussi complexe que la réalité régionale suppose des entrées multiples, parmi lesquelles l'entrée par les flux est la plus fréquemment retenue, parce que le monde contemporain fonctionne sur des interdépendances spatiales croissantes et qu'une entrée par les flux renseigne à la fois sur les interactions et les hiérarchies entre les lieux. Ce n'est cependant pas cette entrée qui est ici retenue, mais celle qui décrit les composantes principales à la base des types d'espaces socio-économiques présents sur le territoire roumain. Depuis 25 ans, les flux n'ont pas eu en Roumanie de priorité fonctionnelle; sous Ceaucescu, les interdépendances ont été plus subies que spontanées et la dynamique du fonds fixe dans les transports et télécommunications a été très inférieure à celle de l'industrie et de la construction (6). Depuis 1990, l'affaissement

(5) La distinction si commode entre "région homogène» et «région polarisée» n'a de sens qu'à un niveau donné d'analyse, car à un niveau supérieur l'homogénéité existe grâce aux interactions associées à la polarisation. Ce qui permet l'identification d'un espace régional par rapport au reste, c'est précisement la plus grande ressemblance des proprićtés à l'intéricur de cet espace qu'à l'extérieur.

(6) Cf. Anuarul statistic al Romaniei, 1990, p. 250, et article de N. Popa dans ce fascicule. général des flux est très spectaculaire, associé à la chute des productions puis à l'explosion des coûts de transport. Par contre, les évolutions du contenu des espaces restent la base de la différenciation du territoire roumain.

\section{Questions, méthodes, mesures}

L'hypothèse qui oriente cette analyse des structures départementales s'inspire de deux durées des processus, et vise à apprécier la part du géographique qui accompagne et façonne l'évolution générale. La première durée prise en considération est celle, somme toute brève, du façonnement socialiste ceaucescien: quelle a été la diffusion spatiale du projet de croissance, doublement fondé sur la mutation du système productif et la dominante industrielle, et sur la généralisation du statut salarial? L'expression spatiale de ce projet de développement socialiste devait se manifester par une complexification des activités et une homogénéisation des conditions de vie. La seconde durée est celle du temps long dans lequel s'inscrivent toutes les architectures spatiales: comment apprécier la part de la différenciation spatiale récente qui revient aux ensembles géographiques naturels de celle qui relève directement de discontinuités introduites par les vicissitudes historiques? En d'autres termes, quelles sont la force de l'étagement des montagnes, des collines et des plaines, et celle de la coupure entre la Transylvanie et le Vieux Royaume, dans la forme d'évolution des structures départementales? Ces questions avaient déjà inspiré le premier travail systématique sur les structures de l'espace roumain au début de la période Ceaucescu (Rey 1973, 1975); elles recoupent partiellement les approches régionales que fournit la littérature géographique roumaine (7).

Les analyses multivariées permettent de dégager les associations de phénomènes ou leurs discontinuités; c'est la

(7) La littérature géographique roumaine sur la question régionale est traversée par le débat entre les tenants de l’analyse régionale fondée en priorité sur des bases physicoorographiques et ceux qui donnent la priorité aux bases fonctionnelles des activités humaines; cf. IaNOS I., 1981, «Puncte de vedere privind analiza geografica regionalð a territoriului Romaniei». Studii si Cercetari geografice, t. XXVIII, p.103-111; cf. POSEA G., 1991, "Geografia generală si regională, reflecţii». Terra, no I, p. 8-12. 
manière la plus opératoire pour mesurer le degré de ressemblance complexe et répondre ainsi à l'un des principes qui fondent la différenciation régionale. Le département (judet) comme niveau d'analyse est le passage obligé pour mobiliser les données disponibles. Effet du souci récurrent d'uniformisation du système socialiste, le maillage des 41 unités est assez régulier, et donc compatible avec l'esprit des traitements statistiques. En 1969, il était le cadre tout neuf d'un maillage artificiel; après 20 ans, les mécanismes d'autocorrélation spatiale à l'intérieur de chaque département ont produit des liens d'interdépendance. Ce qui en 1969 pouvait passer pour une structure spatiale d'inventaire correspond en 1989 à une certaine structure spatiale fonctionnelle, celle qui précisément clôt la période Ceaucescu $(8,9)$.

Du choix des variables dépend le contenu des structures spatiales dégagées et l'orientation de l'interprétation. Ce choix est donc en rapport étroit avec la problématique et les hypothèses retenues. Nous avons sélectionné 15 indicateurs fondamentaux, de même définition à chaque date (tabl. I). Ils recouvrent trois catégories d'information et de processus. Le premier groupe, relatif aux modes de peuplement et aux physionomies spatiales, caractérise la trame minimale sur laquelle portent les équipements et le fonctionnement social et économique concret et sur laquelle s'inscrivent les évolutions générales (croissance, déclin, spécialisation...); ces variables intègrent le double héritage des rapports société-milieu (niveau de densité) et des épisodes historiques (forme du semis de peuplement). Le deuxième groupe d'indicateurs traite des situations démographiques; leur signification prend une importance spécifique dans une aire où la transition démographique est à l'œuvre (taux de natalité de $34 \%$ en 1930 et de $16 \%$ en 1989): or les comportements culturels marquent une capacité à interpréter et

(8) Pour avoir le même maillage aux deux dates, nous avons reconstitué arbitrairement les données de 1969 pour les 3 départements de Giurgiu, Călăraşi et Ialomiţa, créés en 1975 sur l'emplacement des anciens départements d'llfov et de Ialomiţa.

(9) Afin d'éviter la distorsion des résultats que provoque une unité géographique trop différente - ici le département-capitale de Bucarest - nous l'avons traité en individu "supplémentaire", affecté à la classe la plus proche de son profil. qui a toujours été la classe «industrialo-urbaine». à réagir qui dépasse largement l'effet de convergence de transition; la localisation des comportements démographiques conduit donc indirectement à repérer d'autres composantes d'identité sociale (10). Le troisième groupe d'indicateurs, relatif aux activités et aux niveaux d'équipement, permet d'aborder le degré de complexité de la société et du cadre de vie, le niveau de modernisation technique et l'action de l'État. Le phénomène central est celui de la salarisation de la population active, avec disparition du modèle du travailleur indépendant et diffusion des rapports de production socialiste. Ce changement social majeur, synonyme de la modernité en général, est ici étroitement associé à la phase socialiste ( $25 \%$ en 1950, $73 \%$ en 1989 de salariés dans la population active) et au poids croissant du salariat industriel (47\% en 1989). L'entreprise est l'unité fonctionnelle centrale du système socialiste, qui accroît son emprise à travers l'agrandissement des unités. Le niveau d'équipement, tel que l'expriment l'implantation téléphonique et le montant des fonds fixes par habitant, recouvre à la fois le potentiel d'ouverture et d'échanges de l'information, l'héritage d'équipements antérieurs et l'action volontariste de l'État en matière de financements; il sera la base éventuelle du démarrage d'une capitalisation postsocialiste. Nous avons exclu les variables spécifiques des milieux physiques et historiques, afin précisément d'évaluer l'effet de ces particularités géographiques sur les données très générales de comportement socio-économique (11).

(10) Exemple: les coefficients de corrélation linéaire entre le taux de population rurale d'une part et, de l'autre, le taux de natalité et le taux de mortalité, ne sont que de 0,4 et 0,01 , ce qui est une indication précieuse sur la très faible dépendance entre le comportement démographique et le mode de peuplement au niveau national.

(11) Cette méthode peut être considérée comme une application de l'écologie factorielle urbaine à l'échelon régional, avec les modifications qu impose la nature de l'objet analysé. C'est pourquoi ne sont pas retenues les variables de minorités nationales ou religieuses: ici elles correspondent à des hétérogénéités spatiales fixées de longuc durée dont il s'agit d'évaluer l'effet encore actuel. Dans les villes contemporaines (type ville «américaine») c'est leur processus de localisation en cours qui permet de comprendre comment se construit l'espace urbain, d'où leur place à titre de variables dans l'analyse factorielle. 


\section{La situation en 1969}

En 1969, la structuration socio-économique départementale du territoire roumain dépendait principalement de trois axes $(68 \%$ de la variance pour les trois facteurs d'une analyse factorielle en composantes principales, avec mesure par distance euclidienne). L'opposition ville-campagne (F1, 39\% de la variance) en est l'ossature, suivie par ce que l'on peut interpréter ici comme le degré de pénétration de la transition démographique, tel que l'exprime l'opposition entre la forte mortalité infantile et la mortalité générale (F2, 17\%) et par la composition par âge des populations, puisqu'en troisième facteur $(12 \%)$ s'opposent les comportements de natalité et de mortalité générale. Cette structuration spatiale des mécanismes socio-économiques en 1969 opère à travers trois grands types de départements.

Le département industriel-urbain (12 cas) se définit par une très forte salarisation industrielle, excluant le tertiaire et rendant la présence agricole négligeable; le niveau élevé des fonds fixes et de l'équipement, la grande taille des entreprises définissent le profil; la transition démographique paraît achevée, avec une natalité et une mortalité infantile très faibles. Presqu'à l'opposé, se trouve le type du département très rural et agricole (14 cas); le fait industriel et urbain n'y existe pas ou bien il est débordé par la pression des campagnes à très haute natalité; la marque salariale est celle des activités tertiaires (d'administration et de services publics) de nature préindustrielle; le bas niveau d'équipement et de vie se lit dans la très forte mortalité infantile; l'aide de l'État n'est pas parvenue.

Le type de département le plus représenté ( 15 cas) se situe en position intermédiaire, avec des combinaisons de caractéristiques plus hétérogènes; la présence urbaine et industrielle est sensible, avec de grandes entreprises, un certain niveau d'équipement (fonds fixes et téléphone) et une mortalité infantile inférieure à la moyenne; les emplois sont nombreux dans la construction; toutefois, c'est par les comportements démographiques et partiellement par la structure d'âge sousjacente que s'individualise le plus nettement ce type, où la natalité est presque aussi modérée que dans le type à nette dominante urbaine, mais où la mortalité générale est élevée.
La distribution géographique de ces trois types sur le territoire roumain n'a rien d'un puzzle. Le type industrielurbain trace un axe intérieur du Hunedoara, «pays noir» et sidérurgique dès avant la guerre, au Prahova, piémont d'exploitation pétrolière également dès avant-guerre, longeant la retombée interne des Carpates sur la cuvette transylvaine; il englobe les deux départements historiquement les plus marqués par le peuplement saxon, Sibiu et Braşov, puis chevauche les Carpates en direction de Bucarest. Le type intermédiaire se dispose en un large bloc autour de l'axe précédent. Le caractère intermédiaire dans le processus de la modernisation est exprimé par cette contiguité, qui recouvre et rassemble des espaces élémentaires apparemment hétérogènes, à forte ou faible densité, en montagne, en colline ou plaine, avec des passés historiques très différents. Le type hyper-rural et agricole, resté hors de la modernisation, ne concerne que les départements de la Plaine roumaine méridionale et de la Moldavie orientale, plus les deux départements transylvains de Bistriţa-Năsăud et Sălaj, toujours restés à l'écart de l'emprise des peuplements saxon et hongrois. Il est inattendu que Iaşi, à l'égal du Dolj, soit dans ce type et non dans le type précédent; au-delà des schématisations introduites par toute procédure de classification, cette affectation d'appartenance suggère une interprétation liée à la position frontalière nouvelle que subit ce département 
depuis 1940, et à son effet plus que probable de marginalisation du développement industriel qui en est résulté.

Comparativement aux résultats obtenus par la matrice graphique et sur un beaucoup plus grand nombre de variables (V. Rey, 1973, 1975), la structuration des phénomènes est semblable pour ce qui est de l'opposition ville-campagne, du niveau industriel-urbain et de l'insertion dans la transition démographique. La typologie spatiale graphique donne toutefois davantage d'importance à la trame urbaine des grands centres, dessinant une diagonale de plus net développement qui calque les axes ferroviaires internationaux. Mais tout particulièrement les réponses par rapport aux deux questions relatives à l'influence des milieux géographiques et historiques dans la dynamique socio-économique sont semblables dans les deux analyses: les plaines occidentales et sud-orientales appartiennent en 1969 à deux types très distincts, reflets d'appartenances historiques séculairement distinctes. La limite historique, qui durant des siècles a suivi le tracé des crêtes carpatiques, ne possède pas de consistance en 1969 tout au moins quand on examine la structuration de ces variables de base.

\section{La sifuation en 1989}

En 1989, et avec les mêmes variables qu'en 1969, la structuration socio-économique des départements ne repose plus tout à fait sur les mêmes facteurs. Le premier principe de séparation tient moins au niveau d'urbanisation du peuplement et à l'opposition ville-campagne qu'à l'intensité et à la nature de l'activité salariale, opposant la très forte salarisation industrielle et générale au salariat agricole $(\mathrm{F} 1,34 \%)$. Le deuxième facteur de différenciation relève de l'intensité
Tableau I.

A. Valeurs moyennes des quinze variables socio-économiques en 1969 et en 1989. B. Profils moyens des trois types socio-économiques de départements en 1989 (CAH).

\begin{tabular}{|c|c|c|c|c|c|c|c|c|c|}
\hline & & $\begin{array}{l}\text { Rurali: } \\
\text { sotion }\end{array}$ & Notolité & Mortalité & $\begin{array}{l}\text { Mortalité } \\
\text { infantle }\end{array}$ & $\begin{array}{l}\text { Salariés } \\
\text { totoux }\end{array}$ & $\begin{array}{l}\text { Soloriés } \\
\text { industrie }\end{array}$ & $\begin{array}{l}\text { Salariés } \\
\text { construction }\end{array}$ & $\begin{array}{l}\text { Solariés } \\
\text { agriculure }\end{array}$ \\
\hline $\bar{A}$ & $\begin{array}{l}\text { Moyenne } \\
\text { nationale }\end{array}$ & 68,9 & 23,9 & 10,3 & 54,9 & 22,2 & 36,7 & 13,3 & 11,9 \\
\hline 1969 & $\begin{array}{l}\text { Coefficient } \\
\text { de variation }\end{array}$ & 18,5 & 13,3 & 14,3 & 19,3 & 32,7 & 31,1 & 39,8 & 71,3 \\
\hline 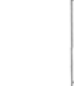 & $\begin{array}{l}\text { Moyenne } \\
\text { nationale }\end{array}$ & 52,1 & 16,5 & 10,8 & 26,7 & 32,5 & 47,4 & 8,5 & 9,4 \\
\hline 1989 & $\begin{array}{l}\text { Coefficient } \\
\text { de variation }\end{array}$ & 23,1 & 11,7 & 14,4 & 27,9 & 16,6 & 18,4 & 36,9 & 62,0 \\
\hline$B$ & $\begin{array}{l}\text { Prépondérance } \\
\text { rurale }\end{array}$ & 56,9 & 18,1 & 9,9 & 26,8 & 28,8 & 49,6 & 7,5 & 8,0 \\
\hline 1989 & $\begin{array}{l}\text { Industriel } \\
\text { et urbain } \\
\text { Fragilité } \\
\text { démographique }\end{array}$ & $\begin{array}{l}40,6 \\
53,3\end{array}$ & 15,1 & 10,3 & 20,1 & 37,8 & $\begin{array}{l}55,5 \\
41,7\end{array}$ & 10,1 & $\begin{array}{r}5,0 \\
13,2\end{array}$ \\
\hline & & $\begin{array}{l}\text { Salariés } \\
\text { tertiaire }\end{array}$ & $\begin{array}{c}\text { Taille } \\
\text { entreprises }\end{array}$ & Densité & $\begin{array}{c}\text { Terres } \\
\text { labourables }\end{array}$ & Téléphone & $\begin{array}{l}\text { Fonds } \\
\text { fixes }\end{array}$ & \multicolumn{2}{|c|}{$\begin{array}{c}\text { Villes } \\
20000 \text { hab. }\end{array}$} \\
\hline $\begin{array}{l}A \\
1969\end{array}$ & $\begin{array}{l}\text { Moyenne } \\
\text { nationale } \\
\text { Coefficient } \\
\text { de variation }\end{array}$ & $\begin{array}{l}38,0 \\
15,0\end{array}$ & $\begin{array}{l}1423 \\
36,3\end{array}$ & $\begin{array}{l}79,1 \\
30,1\end{array}$ & $\begin{array}{l}42,4 \\
51,2\end{array}$ & $\begin{array}{l}11,2 \\
50,9\end{array}$ & $\begin{array}{r}24833 \\
44,9\end{array}$ & $\begin{array}{r}1,9 \\
64,3\end{array}$ & 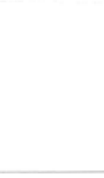 \\
\hline 1989 & $\begin{array}{l}\text { Moyenne } \\
\text { nationale } \\
\text { Coefficient } \\
\text { de variation }\end{array}$ & $\begin{array}{r}34,7 \\
9,3 \\
\end{array}$ & $\begin{array}{l}1593 \\
26,5\end{array}$ & $\begin{array}{l}90,1 \\
32,2 \\
\end{array}$ & $\begin{array}{l}40,7 \\
52,8\end{array}$ & $\begin{array}{l}77,9 \\
27,1\end{array}$ & $\begin{array}{c}134630 \\
38,3\end{array}$ & $\begin{array}{l}2,7 \\
7,0\end{array}$ & \\
\hline$B$ & $\begin{array}{l}\text { Prépondérance } \\
\text { rurale }\end{array}$ & 35,5 & 1588 & 92,6 & 35,2 & 73,2 & 106612 & 2,4 & \\
\hline 1989 & $\begin{array}{l}\text { Industriel } \\
\text { et urbain }\end{array}$ & 32,5 & 1937 & 91,2 & 22,5 & 95,4 & 137631 & 4,5 & \\
\hline & $\begin{array}{l}\text { Fragilité } \\
\text { démographique }\end{array}$ & 35,0 & 1435 & 87,5 & 54,2 & 73,9 & 157941 & 2,0 & \\
\hline
\end{tabular}

de l'action de l'État en matière d'investissement et d'équipement (F2, 20\%). Le troisième facteur reste celui des comportements démographiques, opposant la mortalité générale à la natalité $(F 3,12 \%)$. Cette comparaison structurelle de deux états successifs à vingt ans d'écart souligne bien ce qui a été expressément la marque ceaucescienne du développement socialiste roumain: une modernisation des structures par un transfert simple au travers d'une industrialisation à outrance, à coups d'investissement d'État, mais non accompagnée d'une véritable complexification des structures d'activités.

Les trois types de départements qui, en 1989, participent à cette structuration des éléments socio-économiques ne sont 


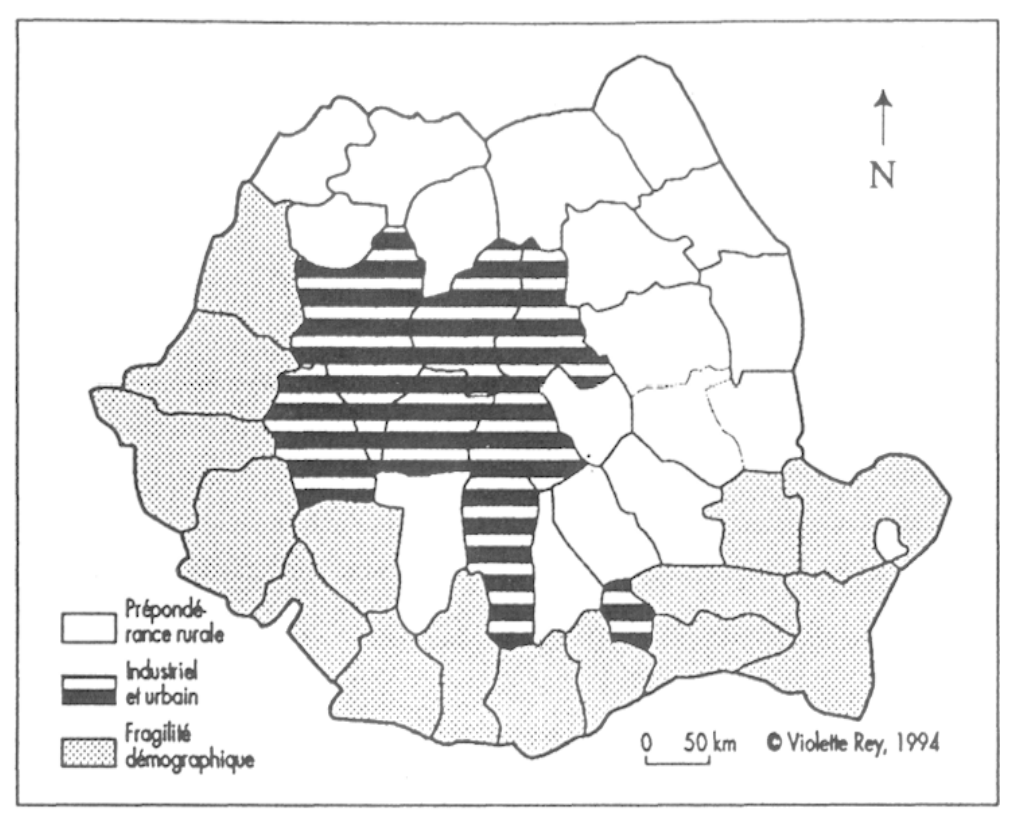

Fig. 2.-Types de structures socio-économiques en 1989. Typologie effectuée par classification ascendante hiérarchique sur 15 indicateurs divers.

est nettement inférieure à la moyenne et dans le contexte des plus modestes densités roumaines. Seul le type industriel-urbain, constitué de 9 cas, reste d'un profil tout à fait comparable au type de 1969, avec une forte salarisation générale et industrielle et des comportements démographiques combinant faible natalité et faible mortalité générale à une très faible mortalité infantile.

La configuration géographique de ces trois types de 1989 (fig. 2), tels qu'on peut les identifier après 20 ans de fonctionnement départemental du système socialiste roumain, est encore moins un puzzle qu'en 1969, puisqu'il s'agit de trois blocs emboîtés: les départements industriels et urbains comme môle central dans la Transylvanie du Centre-Sud; les départements intermédiaires ayant conservé une natalité et une ruralité spécifiques recouvrent toute la Moldavie, le piémont munténien et la Transylvanie du Nord; les départements que l'on peut définir en état de tension

pas non plus similaires aux types dégagés en 1969. Le plus représenté (17 cas) a un caractère intermédiaire dans la mesure où le taux général de salariés et celui des salariés industriels ont une surreprésentation modérée par rapport à la moyenne, mais non associée à un état d'équipement et à un effort d'investissement de même niveau. Ce type de département se singularise par un haut niveau de peuplement rural et presque toujours par une forte densité de population, accompagnés d'une natalité très supérieure à la moyenne de 1989. On constate ainsi le changement de rôle que tient la natalité dans la différenciation spatiale entre les deux dates. En 1969, juste après la loi anti-avortement qui avait provoqué un pic de naissances, le caractère modéré de la natalité était lié à une certaine présence urbaine et industrielle et pouvait s'interpréter comme synonyme d'un processus plus global de modernisation des comportements dans les départements où cette association était observée. En 1989, c'est au contraire le caractère de forte natalité (relativement à cette date) associé à la présence de forte densité et de forte ruralité, qui spécifie le type de département intermédiaire. La singularisation spatiale par les comportements démographiques définit également de façon tranchée un deuxième type départemental, représenté par 15 cas, où la surreprésentation de la mortalité générale et de la mortalité infantile vont de pair, tandis que la natalité y démographique forment un arc périphérique des plaines occidentales du Crisana-Banat à la Plaine roumaine, jusqu'à la Dobroudja et à la mer Noire (12).

Cet état structurel de 1989 ne manque pas de surprendre par les associations globales qui y sont révélées, sans pour autant valider la force des deux principes structurants pris comme hypothèses, celui des milieux physiques et celui de la trace historique. Plus encore qu'en 1969, ce sont les situations démographiques définies par les comportements face à la vie (et partiellement sous-tendus par le degré de jeunesse ou de vieillissement), qui différencient l'espace socio-économique roumain pris dans son ensemble; davantage que la

(12) Peuvent paraitre mal classés le Prahova et le Covasna, hors du bloc industrialo-urbain auquel on penserait de prime abord qu'ils appartiennent. L'affectation du Prahova au type intermédiaire tient à sa combinaison toute particulière d'un très haut niveau de salariat industriel $(57 \%)$ et d'un milieu où la ruralité est égale à la présence urbaine. La séparation du duo Covasna-Harghita, les deux départements montagnards à peuplement hongrois, reflète en Covasna une natalité plus élevée et surtout une mortalité infantile surprenante pour la Transylvanie centrale ( $26 \%$ contre $15 \%$ en Harghita); est-ce imputable à l'ethnicité sicule au sein du rameau hongrois? 
volonté industrialisante qui a diffusé partout, comme si l'expression sociale l'emportait sur l'expression étaticoéconomique dans le marquage spatial. Si montagnes et collines ne se détachent pas, le phénomène géographique de la plaine acquiert une individualisation humaine nouvelle, au point d'associer la plaine occidentale et la plaine méridionale historiquement séparées. Le trait commun en est cette tension démographique, qui met en relief d'autres propriétés communes de leur peuplement, de densité moindre et d'âge d'implantation récent. Enfin, la séparation historique entre la Transylvanie et le Vieux Royaume n'est pas plus lisible qu'en 1969, même si, parallèlement, l'individualisation de la Transylvanie du Sud et celle des pays roumains de Moldavie et Maramureş paraît plus nette.

\section{Les trajectoires déparfementales}

Parallèlement à l'analyse des états successifs, nous avons évalué la dynamique propre à chaque département en classant sa trajectoire au sein de l'évolution des variables (13, 14). En général, dans ce genre d'analyse, les types spatiaux identifiés sont représentés aux deux périodes et les trajectoires

(13) La classification est établie sur le tableau composé des 16 variables et des 80 unités géographiques que sont les départements en 1969 puis en 1989. Par référence à une structure moyenne théorique, qui représente un état intermédiaire entre 1969 et 1989, la CAH définit des types de structures spatiales qui peuvent n'exister qu'à l'une ou l'autre des deux dates ou aux deux, et chaque département peut soit conserver le même profil soit en changer. Cette méthode permet assez explicitement de définir le sens de la dynamique générale à travers la prise en compte de toutes les dynamiques individuelles: en fin de période, il y a convergence, donc homogénéisation spatiale, si les départements se répartissent en un nombre réduit de types, ou au contraire divergence et différenciation spatiale accrue si le nombre de types représenté est supérieur à celui de la situation initiale.

(14) Le niveau de dispersion des valeurs de chaque variable, mesuré par le coefficient de variation à chaque date, permet de distinguer les variables dont la discrimination spatiale est élevée et stable (labour, téléphone, densité), celles qui contribuent à l'homogénéisation au cours de la période parce que leur coefficient de variation se réduit (salariés, salariés industriels, fonds fixes), celles qui deviennent plus différenciatrices, comme la mortalité infantile dont le coefficient passe de 19 à 27,5 entre 1969 et 1989. alors que parallèlement la mortalité infantile décroît de $55 \%$ à $27 \%$. des unités géographiques dessinent des orientations variées. Or, dans le cas de l'espace roumain entre 1969 et 1989. l'ampleur du passage en bloc d'un état de ruralité (certes déjà entamé par les changements des décennies 1950-1960) à une certaine modernité conduit à une quasi totale mutation du contenu structurel: 37 départements sur 41 ont en 1989 des profils qui n'existaient pas en 1969; 3 des 4 types identifiés en 1969 ont disparu en 1989!

Que ce soit par les variables démographiques et par les taux d'emploi dans la construction et le tertiaire, qui déclinent dans tous les départements, ou par les variables de la modernisation socialiste exprimée par le salariat, la grande industrie et les investissements, qui progressent dans tous les départements, l'ampleur du changement des valeurs des variables fait que la ressemblance interne des départements à chaque date est plus élevée que les ressemblances que certains départements auraient pu conserver au cours du temps. On peut considérer que cette forme du changement témoigne d'une remarquable réussite apparente de l'objectif de l'État, réduire les écarts majeurs entre les villes et les campagnes, entre les régions arriérées et les régions développées, grâce à la diffusion du salariat industriel. On peut aussi pressentir ce que cela signifie d'effort artificiel pour aboutir, en vingt ans seulement, à un résultat aussi spectaculaire et rare dans les annales du changement des structures spatiales.

Compte tenu du caractère assez répétitif des analyses, nous nous limitons à donner le tableau croisé des types aux deux dates (tabl. II), établi à partir de la classification dite des trajectoires, et nous soulignons la position très particulière des trois cas d'espèce que sont les départements de Braşov, Sibiu et Hunedoara. Dès 1969, ils sont du type industrielurbain, parce que dès cette date ils combinent de façon très marquée deux propriétés, l'une relative au mode de peuplement, l'autre à l'activité: une surreprésentation des villes de plus de 20000 habitants et une sous-représentation de la population rurale; une surreprésentation du salariat général, du salariat industriel et des grandes entreprises. Se dessine donc en 1969, dans ces vieux foyers industriels et urbains de la Transylvanie méridionnale, ce qui deviendra le modèle du contenu départemental moderne et socialiste. Mais l'absence d'apparition en 1989, dans l'un ou l'autre de ces départements, d'un type de profil spatial plus évolué et complexe qu'en 1969 (en particulier avec une émergence 
Tableau II

Types socio-économiques et trajectoires des départements roumains entre 1969 et 1989.

\begin{tabular}{|c|c|c|c|c|}
\hline 1989 & $\begin{array}{c}\text { Prépondérance } \\
\text { rurale }\end{array}$ & $\begin{array}{l}\text { Industriel } \\
\text { et urbain }\end{array}$ & $\begin{array}{c}\text { Fragilité } \\
\text { démographique }\end{array}$ & $\begin{array}{l}\text { Nombre de } \\
\text { départements par } \\
\text { classe en } 1969\end{array}$ \\
\hline $\begin{array}{l}\text { Régions } \\
\text { montagneuses }\end{array}$ & $\begin{array}{l}\text { Satu Mare } \\
\text { Sălaj } \\
\text { Maramures } \\
\text { Bistrita-Nasaud } \\
\text { Neamt } \\
\text { Covasna } \\
\text { Vîlcea } \\
\text { Vrancea } \\
\text { Suceava }\end{array}$ & $\begin{array}{l}\text { Alba } \\
\text { Harghita }\end{array}$ & $\begin{array}{c}\text { Gorj } \\
\text { Caras-Severin } \\
\text { Mehedinți } \\
\text { Dimbovița }\end{array}$ & 15 \\
\hline $\begin{array}{l}\text { Régions } \\
\text { agricoles }\end{array}$ & $\begin{array}{c}\text { Botossani } \\
\text { lași } \\
\text { Vaslui } \\
\text { Galati } \\
\text { Buzău }\end{array}$ & & $\begin{array}{c}\text { Tulcea } \\
\text { Braila } \\
\text { lalomița } \\
\text { Calarași } \\
\text { Giurgiu } \\
\text { Doli } \\
\text { Olt } \\
\text { Teleorman } \\
\text { Constanța }\end{array}$ & 14 \\
\hline $\begin{array}{l}\text { Régions } \\
\text { d'économie } \\
\text { moderne }\end{array}$ & Bacău & $\begin{array}{c}\text { Bucarest } \\
\text { Cluj } \\
\text { Mures } \\
\text { Brasov } \\
\text { Sibiu } \\
\text { Arges }\end{array}$ & $\begin{array}{l}\text { Prahova } \\
\text { Arad } \\
\text { Timis } \\
\text { Bihor }\end{array}$ & 11 \\
\hline \multicolumn{2}{|c|}{$\begin{array}{l}\text { Nombre de } \\
\text { départements } \\
\text { par classe en } 1989\end{array}$} & 8 & 17 & 40 \\
\hline
\end{tabular}

chaque État offre une physionomie du changement très particulière. La Roumanie n'a connu ni guerre, ni partition politique, ni nette élimination de la classe politique précédente, mais comme ailleurs effondrement économique, crise sociale et tâtonnements vers l'économie de marché. Certaines décisions apparaissent toutefois comme des réponses spécifiques du système national. On devine l'intérêt qu'il y a à localiser ces phénomènes nouveaux, afin d'apprécier dans quelle mesure ils s'inscrivent dans la dynamique spatiale précédente, et dans quelle mesure ils éclairent le regain d'attitude qui consiste à s'identifier selon une préférence provinciale historique.

\section{Types spatiaux en début de transition}

Pour ce coup de sonde sur la mise en place de la transition roumaine, nous avons retenu ce qui en parait le plus spécifique, à savoir les variations démographiques comme première expression de la liberté recouvrée des individus et des

d'activités tertiaires) en dit long sur le blocage général du modèle de développement ceaucescien, quand on l'observe à l'aune de ses résultats spatiaux.

\section{Lieux de transition et incertitudes des recompositions régionales}

C'est sur cet espace roumain structurellement affecté de modifications si considérables et si peu conformes aux caractères usuels de la modernisation spatiale, que s'est propagé le choc de la chute de Ceaucescu, puis le processsus de la désocialisation. Face à l'éclatement du second cercle soviétique et aux problèmes très généraux dits de la transition, même si certaines décisions sont automatiquement semblables, familles, le changement économique à travers l'apparition du chômage et des activités privées, et l'ouverture sur l'étranger à partir des équipements de télécommunication (15).

(15) Aucun indicateur sur les variations de valcurs de productions (industrielles ou agricoles) n'a été retenu, soit que la désagrégation spatiale dépende trop du statut juridique des entreprises et ne restitue pas encore l'image des lieux de production, soit que l'estimation en paraisse trop incertaine. Quant aux sociétés mixtes. leur très faible volume et leur surconcentration à Bucarest (plus de $60 \%$ ) indiquent que leur insertion dans la dynamique régionale est encore négligeable mais touchc l'axc Timişoara, Sibiu,Tirgu-Mureş, puis Braşov, Bucarest. Cf. Ianoş I., GuRan L., (1993). «Regionale Aspekte ausländischer Investitionen in Rumänien seit 1990», Österreichische Hefte, vol. 35, n 4, p. 633-648. 


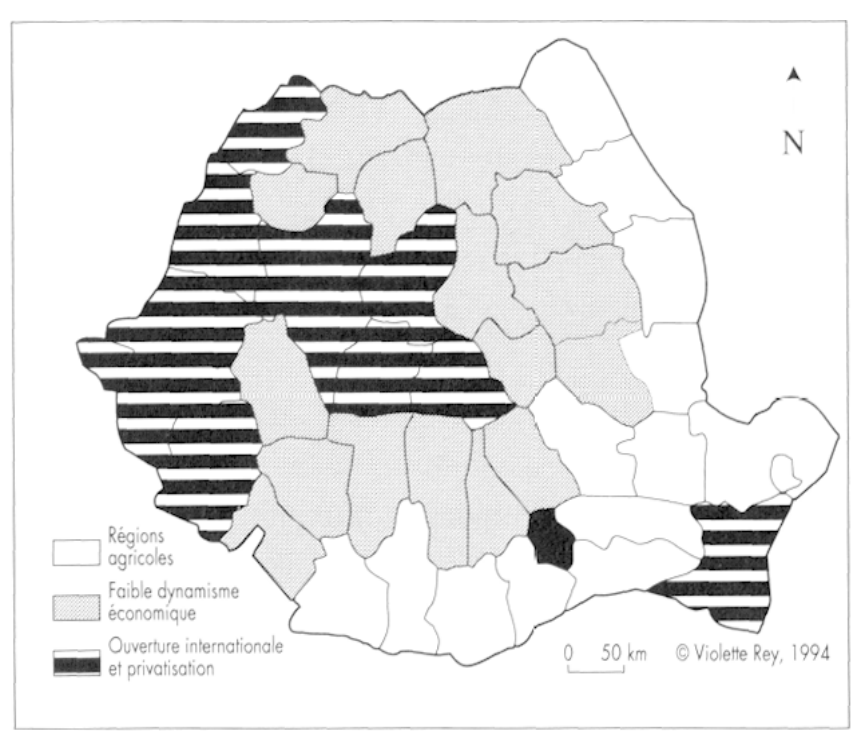

Fig. 3.-Types de transition en 1993.

Typologie effectuée par une classification ascendante hiérarchique avec 7 indicateurs.

La vigueur de la perturbation démographique est à la mesure du choc historique qu'est la chute du système: en trois ans (1989-1992), la population totale diminue de 420000 habitants et la natalité tombe de $16 \%$ à $11,4 \%$. Cette diminution de population est d'abord due à la possiblité de quitter le pays, d'émigrer durablement ou même définitivement, ce que choisissent la majorité des Allemands encore en place; d'où l'effet spécifique de baisse de population dans les régions méridionales de Transylvanie et du Banat, et dans les villes dont une partie des cadres tente l'aventure à l'Ouest. Elle tient aussi au brutal affaissement de l'apport des naissances (260 000 contre 370000 ), alors que le niveau de mortalité a tendance à augmenter pendant ces mêmes trois années (264 000 contre 247000 ). On sait le rôle tenu dans ce phénomène par la relibéralisation du droit à l'avortement, décidée dès janvier 1990, dont la géographie très différenciée révèle des comportements sociaux opposés. À l'exception du département urbain de Iaşi, la Moldavie et la Transylvanie du Nord, toujours caractérisées par un haut niveau de natalité, ne pratiquent guère l'interruption de grossesse; pas davantage les régions occidentales du Crisana et du Banat, dont les taux de natalité sont les plus bas; par contre, tout le quart sud-est du pays (plaine du Bărăgan, Dobroudja et même piémont munténien), où le niveau de natalité en 1989 était en recul, adopte ce mode de limitation de naissances qui accentue le ralentissement naturel du niveau de peuplement.

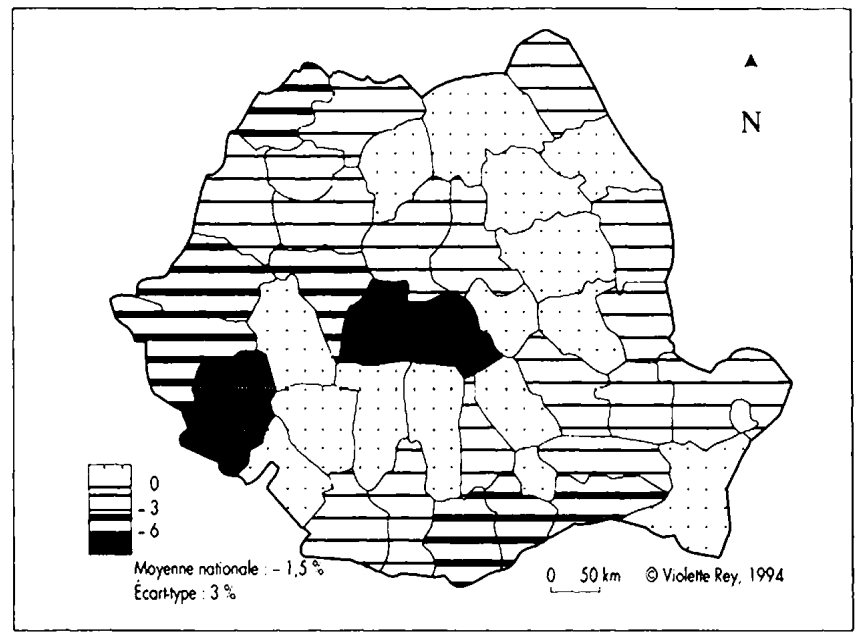

Fig. 3.1.-Déclin de la population 1989-1992.

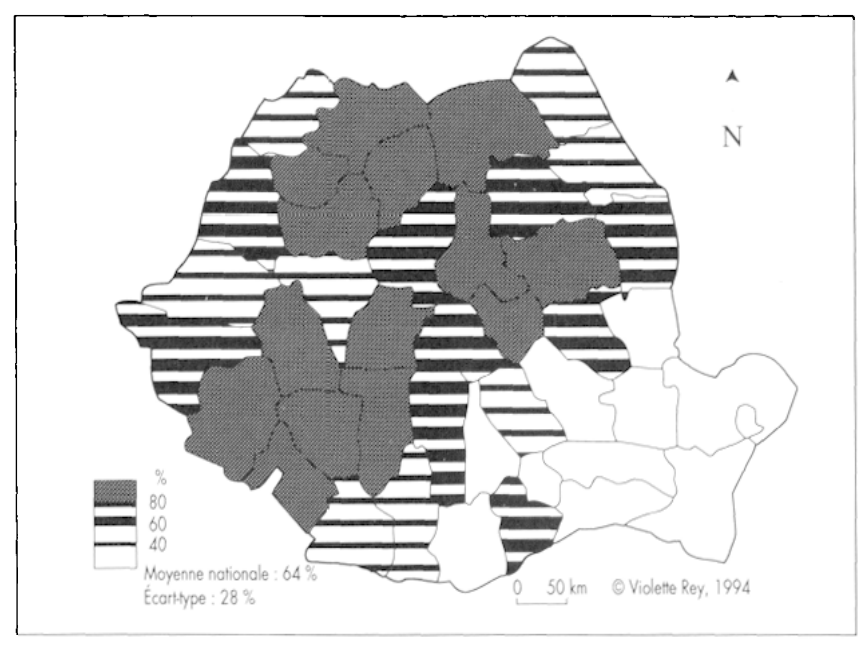

Fig.3.1.- Superficie agricole travaillée individuellement en 1992.

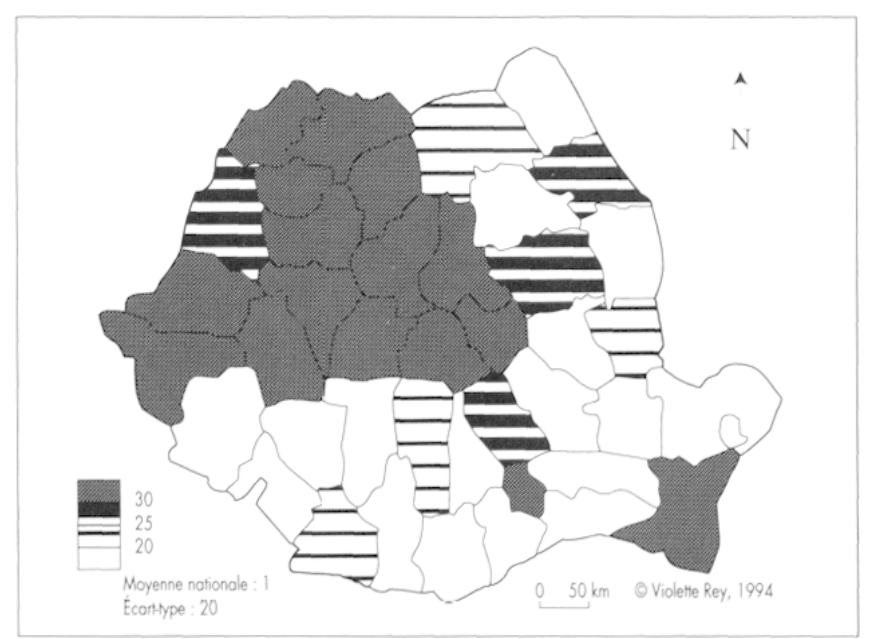

Fig. 3.1.-Nombre d' abonnements télex pour 10000 habitants en 1992 
La reprivatisation de l'agriculture est assurément le phénomène le plus original que la Roumanie apporte au processus général de la transition postsocialiste (B. von Hirschhausen, 1994); la disparition des grandes unités coopératives de production et le retour du travail paysan individuel donnent naissance à un nouveau paysage de champs étroitement laniérés, qui avait totalement disparu du paysage socialiste. Fin 1991, le travail hors des associations touche $65 \%$ de la superficie agricole, avec deux pôles montagnards où le phénomène est redevenu exclusif: les Carpates du Sud-Est avec le piémont olténien, les Carpates du Nord, du Maramureş à la Moldavie-Bucovine. Mais cette reprivatisation est presque inexistante dans le quart sud-est, de Galaţi et Buzău jusqu'au Teleorman, dans ces plaines de Bărăgan et Dobroudja où la présence des fermes d'État a toujours été la plus forte et où, probablement, demeurera une agriculture de grande entreprise.

Comme dans tous les anciens pays socialistes, la petite privatisation touche les divers commerces de détail (par privatisation des unités socialistes antérieures ou par création de fonds de commerce indépendants); elle représente $60 \% \mathrm{du}$ nombre des unités commerciales fin 1992, avec une localisation en partie inattendue, parce que concentrée à $62 \%$ dans 12 départements sur 41 . Qu'aux côtés de la capitale (21\% du total) on trouve le département du port de Constanţa et les départements très industriels de Galaţi, Braşov, Sibiu et des piémonts se conçoit; mais que ni le Banat, ni Cluj ni Iaşi n'apparaissent dans ce groupe laisse perplexe: doit-on le lire comme une floraison des microcommerces dans les pays de collines de Moldavie et Valachie, tandis qu'au centre et à l'ouest du pays la nouvelle organisation commerciale privée (qui existe tout autant) se structure autrement, autour d'unités moins nombreuses? Cette différence est confirmée par la localisation des abonnements de télex, nombreux dans l'aire transylvaine et occidentale, à Bucarest et Constanţa. La répartition des contacts internationaux téléphoniques, signe distinctif des activités urbaines, dessine la géographie des grandes villes et de l'axe transylvain, avec toutefois en position de retrait Dolj (Craiova), Galaţi, Brăila, et même Prahova dont on retrouve le déclassement dans la modernité observé en 1989. L'apparition du chômage $(8,4 \%$ officiellement fin 1992) sur les décombres du système productif socialiste affecte surtout le tiers oriental du pays, où la population jeune est abondante et où l'ouverture à l'économie industrielle a été tardive (p. 344, fig. 2, in Popescu). Ailleurs, les constrastes sont sensibles d'un département à l'autre selon le maintien ou non des commandes en usines. On touche là à cette régularité d'association géographique entre le chômage et le bas niveau du développement matériel que n'a pu casser le système socialiste.

Ainsi, la répartition géographique de caractéristiques propres au début de la transition (16) s'avère d'emblée significative. Des similitudes de proximité et les semis en agrégats l'emportent dès la mise en route du changement. La nature fragmentée de l'émergence de la transition se révèle aux faibles niveaux de corrélation des répartitions spatiales. La corrélation la plus élevée porte sur la reprivatisation des terres agricoles avec la natalité en 1992 $(r=0,4)$ et avec sa variation conjoncturelle $(r=0,5)$ : signe d'attitudes nouvelles à l'égard des modes de vie futurs, peut-être corroboré par la corrélation faible mais de signe négatif avec le commerce privé $(r=-0,26)$. Par contre le taux de chômage, déjà peu corrélé au niveau de natalité $(0,04)$, est sans relation avec l'attitude de réduction des naissances ou avec la reprise de l'agriculture familiale $(r=0,00)$.

Une typologie des départements selon leurs profils de transition fait apparaître une coupure étonnament significative de part et d'autre de la ligne de crêtes des Carpates, et met en relief le double croissant des départements de piémonts et des départements des plaines périphériques sud-orientales. Comme si, dès la fin de la pression totalitaire en faveur d'un arrangement spatial uniforme, la société roumaine réagissait en remobilisant les milieux géographiques les plus proches des grands ensembles naturels qui, à peine voilés par l'énorme effort de modernisation, reprenaient

(16) Les 7 variables retenues combinent états de situation et variations sur une très courte période, ce qui autorise à les considérer toutes comme variables d'état de transition. Il s'agit de: taux de variation de la population totale 1989-1992, taux de variation de la natalité 1989-1992, \% de terre agricole travaillée individuellement en 1992, \% d'unités commerciales privées dans le total des unités commerciales, \% de chômcurs dans la population active, nombre d'abonnements télex pour 1000 habitants, $\%$ de communications internationales dans le total des appels téléphoniques, tabl. III. 
Tableau III

Types spatiaux de la transition en Roumanie en 1993

(tableau des profils moyens).

\begin{tabular}{|c|c|c|c|c|c|c|c|}
\hline $\begin{array}{l}\text { Type } \\
\text { spatial }\end{array}$ & $\begin{array}{l}\text { Variation } \\
\text { de la } \\
\text { population }\end{array}$ & $\begin{array}{c}\text { Variation } \\
\text { de la } \\
\text { natalitié }\end{array}$ & $\begin{array}{l}\text { Surf. agric. } \\
\text { exploitées } \\
\text { en individuel }\end{array}$ & $\begin{array}{l}\text { Commerces } \\
\text { privés }\end{array}$ & Chômage & $\begin{array}{l}\text { Abonne- } \\
\text { ments télégra- } \\
\text { phiques }\end{array}$ & $\begin{array}{c}\text { Communica- } \\
\text { tions interna- } \\
\text { tionales }\end{array}$ \\
\hline Bucarest & 1,0 & $-33,1$ & 45,9 & 82,6 & 4,9 & 1,44 & 0,26 \\
\hline $\begin{array}{l}\text { Ouverture } \\
\text { économique } \\
\text { internationale }\end{array}$ & $-4,5$ & $-26,7$ & 66,3 & 55,2 & 7,2 & 0,11 & 0,03 \\
\hline $\begin{array}{l}\text { Dominante } \\
\text { agricole }\end{array}$ & 0,4 & $-26,2$ & 86,5 & 48,7 & 8,9 & 0,05 & 0,01 \\
\hline $\begin{array}{l}\text { Faible } \\
\text { dynamisme } \\
\text { économique }\end{array}$ & $-1,8$ & $-31,7$ & 38,1 & 54,7 & 11,0 & 0,03 & 0,01 \\
\hline $\begin{array}{l}\text { Moyenne } \\
\text { nationale }\end{array}$ & $-1,6$ & $-28,2$ & 64,7 & 53,2 & 9,0 & 0,09 & 0,02 \\
\hline
\end{tabular}

pleinement leur avantage architectonique fondamental! II était difficile d'imaginer une telle ampleur de l'effet des milieux écologiques sur la combinaison de variables élémentaires, même si l'observation sur le terrain en suggérait un ceratin impact. À ces trois ensembles naturels correspondent trois réactions initiales à la transition. L'ouverture à l'horizon ouest-européen, qui suscite aussi bien les contacts de télécommunication que les départs d'émigrants mais épargne du chômage, est propre à la Transylvanie et au Banat-Crisana. La réappropriation immédiate et totale de l'espace agraire et le maintien démographique sont caractéristiques des collines et des montagnes, foyers constants du ressourcement de l'âme roumaine, lieux de toutes les richesses naturelles traditionnelles et si fortement présentes dans la poésie. La transition comme retour du chômage, multiplication des petits métiers et maintien de la grande agriculture marque les plaines périphériques, où le peuplement généralisé a été tardif et où la dépopulation réapparaît dans les villages. Ce caractère structurellement non aléatoire de la géographie du début de la transition ne me paraît pas devoir être considéré comme une chose allant de soi; on auraient mieux compris que les premières manifestations fussent spontanément dans le désordre spatial, au moins dans une saisie du territoire national à l'échelle grossière des départements.
2. Vers une recomposition en grands ensembles régionaux

L'analyse diachronique a fourni la charpente des combinaisons socio-économiques qui structurent le territoire roumain; l'autre entrée analytique porte sur les liens fonctionnels d'interdépendance entre les lieux. Par l'articulation entre ces deux approches il est possible de saisir les cas où les similitudes physionomiques sous-tendent des complémentarités, et ceux où l'emportent des interactions réductrices des différences.

Les flux migratoires, selon le premier bilan départemental qu'en a dressé le Service statistique roumain (17), donnent une image très contrastée des interdépendances régionales. En ne retenant que les flux supérieurs à 10000 personnes, l'effet banal de proximité est atténué au profit des interdépendances lointaines entre régions à surplus démographique et régions manquant de main-d'œuvre, avec les éventuelles préférences régionales qui filtrent de tels mouvements. La Moldavie, à forte pression démographique, émet de toutes parts; par contre Bucarest, les grands centres industriels et le Banat sont attractifs de façon très différentielle. La forme et l'extension du réseau d'attraction de la capitale s'appuie exclusivement sur ce qui fut le territoire du Vieux Royaume, plus les deux antennes de Braşov et de Cluj sur la diagonale de plus haut niveau de développement. Constanţa aspire les surplus moldaves dans un large rayon, contrastant avec l'attraction de voisinage

(17) Il s'agit d'une approche sommaire, déduite des données du recensement de 1992, des flux entre départements lieux de naissance et départements lieux de résidence, tous âges confondus. Demografia, Bucarest 1994. 


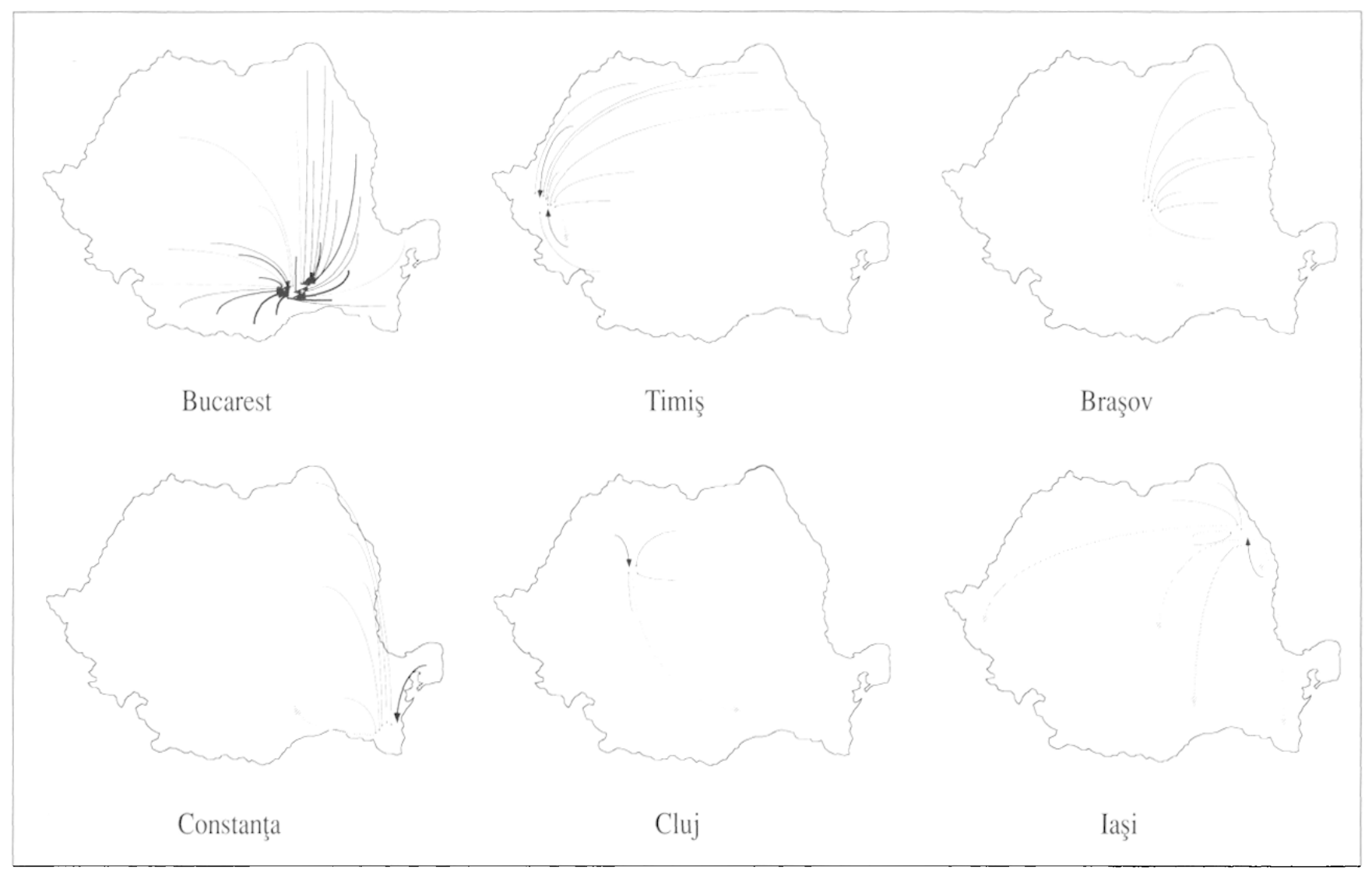

Fig. 4.- Les départements ayant la plus forte attraction migratoire.

observée pour Galaţi. Les flux migratoires dans la Transylvanie intérieure sont modérés et locaux, favorisant l'interconnaissance intrarégionale, comme le montrent les cas de Cluj, de Mureş et de Sibiu, excepté Braşov qui reçoit des migrants de toute la Moldavie mais sans mordre sur la Transylvanie centrale, comme si ce département avait perdu ses attaches historiques. Le Banat, fort demandeur de main-d'œuvre, recrute sur son aire provinciale historique du Crisana et des couloirs du Mureş et du Danube, dans la lointaine Moldavie, mais sans davantage pénétrer dans la Transylvanie du Centre-Nord.

Le raccordement de ces figures migratoires aux figures structurelles précédemment établies suggère certaines réflexions sur les lieux de discontinuité, les lieux de polarisation et les aires intermédiaires. Cela conduit à réévaluer les hypothèses du poids de la division historique ancienne et du poids des milieux géographiques locaux dans la dynamique contemporaine soumise au retour des affirmations identitaires et à l'ouverture sur de nouveaux champs de force internationaux postsocialistes.
L'irruption de nouveaux champs de force internationaux, ceux de la démocratie parlementaire et de l'économie marchande réactive le gradient de la modernité occidentale, objectivement propice au rajeunissement de la coupure historique. Repérée dans l'ordre du matériel sur la carte des types de début de transition, cette coupure n'est pas aussi claire dans l'approche de l'identité symbolique telle qu'on peut indirectement la saisir à travers les comportements politiques et les résultats électoraux. Au premier vote libre de 1990, encore sous le choc des violences et de la confusion des événements qui suivent l'élimination de Ceaucescu, seuls les trois départements à très forte composante hongroise échappent à l'envol du Front du salut national: Harghita, Covasna et Mureş (moins de 20\%) (18); mais les taux quasi plébiciscitaires, supérieurs à $80 \%$, n'apparaissent que

(18) Recensement de 1992: la population de nationalité hongroise est de 1,62 million d'habitants (soit 7,1\% du total) dont $44 \%$ résident dans les trois départements du Harghita (79\% de la population totale locale), du Covasna (74\%) et du Mureş ( $41 \%$ ). 
dans la Plaine roumaine et le bas Danube. Aux votes de 1992, quand a pris place une diversité des partis, les attitudes nationalistes débordent de la Transylvanie centrale sur les piémonts mais sans toucher le Banat et, surtout, les partis opposés au pouvoir en place (Convention démocratique et parti de P. Roman) atteignent des scores significatifs sur une large bande allant du Banat à Constanţa, et touchant l'Olténie et la Moldavie centrale. Sachant en d'autres pays la versatilité du choix des électeurs pour des partis aux programmes peu crédibles, ces résultats sont ici retenus pour l'information spatiale qu'ils transmettent à propos d'attitudes politiques similaires, plutôt que pour leur signification politique intrinsèque. Plus révélatrice encore a été la coupure entre le vote des villes et le vote des campagnes, qui a aussitôt remis en lumière l'affrontement central qui divise la société roumaine depuis le $\mathrm{XIX}^{\mathrm{C}}$ siècle.

Ces décalages entre les identifications symboliques affirmées (appartenance à la Transylvanie, la Moldavie, la Munténie, l'Olténie) et les analyses systématiques laissent certaines appartenances géographiques indécises. La Transylvanie à l'ouest de la ligne des Carpates ne constitue pas un seul ensemble, même si la coupure historique retrouve plus de lisibilité. La Transylvanie méridionale, fortement intégrée, ayant beaucoup de liens avec Bucarest et la Munténie proches, mais touchée de plein fouet par le délestage de la population allemande, est devenue de plus en plus différente de la Transylvanie du Centre-Nord (19). L'analyse structurelle a ici identifié dès 1969 le type socialiste moderne, issu d'un greffon sur un tissu industriel et urbain plus ancien; ce type s'est ensuite figé dans la structure acquise. C'est pourquoi la Transylvanie méridionale, cette partie la plus intérieure et centrale du territoire roumain, dévoile une fragilité nouvelle; deviendra-t-elle une entité régionale distincte, charnière entre la Transylvanie Nord et la Munténie? Un certain rattachement à l'une ou à l'autre l'emportera-t-il? La question est à approfondir également sur la bordure pannonienne: le Banat affirme son statut de région pannonienne ouverte (prémices d'un aéroport international) mais dont les bases démographiques défaillantes sont toujours à renouveler. La ressemblance structurelle s'est renforcée du

(19) Cetle coupure intratransylvaine renvoic à celle imposéc pendant la seconde guerre mondiale. quand seule la partic septentrionale fut rattachée à la Hongrie.
Bihor au Timişoara entre 1969 et 1989 et le comportement du début de la transition y est semblable. Cependant, d'autres éléments de l'identité et du fonctionnement sont nécessaires pour évaluer dans quelle mesure les régions d'Oradea et de Satu Mare participent davantage de l'aire nord-transylvaine ou de l'aire pannonienne et banatique.

La vitalité des axes internationaux de circulation VienneIstanoul ira sans doute dans le sens d'une articulation NOSE plutôt que N-S. La réarticulation de toute la partie orientale et méridionale du pays dépendra probablement aussi des possibilités d'ouverture internationales. La question de l'émergence d'un ensemble régional des bouches du Danube-mer Noire est posée: la vigueur de l'ouverture maritime de Constanţa n'a cessé de s'affirmer entre 1969 et 1989, puis de se confirmer en début de transition. Mais, à cause de la coupure qu'introduit l'immense cours du bas Danube s'agit-il d'autre chose qu'une apophyse de la Dobroudja, reliée au centre par le canal Danube-mer Noire? Dans quelle mesure Galaţi, historiquement moldave, s'y rattachera-t-elle? Depuis 25 ans, les similitudes physionomiques ont consolidé du Bărăgan à la Dobroudja une aire d'homogénéité à laquelle participe partiellement le département de Galaţi. Sur cette aire d'interférence ou de marge, le futur de l'appartenance régionale est indécis. Enfin, compte tenu du poids de la capitale au sein de la population du pays $(20 \%)$ et dans le semis des métropoles européennes de plus de 2 millions d'habitants, compte tenu de sa fonction renforcée de relais pour la mise en réseau de l'ensemble du pays avec l'extérieur (plus de $60 \%$ des sociétés mixtes), beaucoup du devenir de chaque région et de l'articulation des régions entre elles passera par Bucarest.

\section{Conclusion}

Deux conclusions principales peuvent être tirées pour l'action sur l'espace territorialisé et la connaissance de l'espace géographique. L'approche socio-économique de la structuration de l'espace roumain a permis de dégager des combinaisons d'interdépendances spécifiques et d'apprécier leurs articulations par rapport aux entrées ethnico-historique et écologique. Face au syndrome industrialiste homogénéisateur, la réaction sociale, exprimée par des comportements démographiques, a fait preuve d'une capacité de différenciation spatiale majeure. Conçus comme une évaluation 
préliminaire des cohésions régionales et des cristallisations des régionalismes, ces résultats sont nécessaires à toute réflexion politique portant sur la réorganisation des collectivités locales et sur une éventuelle réforme administrative.

Faut-il que tout change pour que tout reste pareil? Cette réflexion que Lampedusa prête à son héros semble s'appliquer à la dynamique récente de l'espace roumain. Ceaucescu a cassé l'émergence d'un axe de développement, qui fut transversal à la double coupure naturelle et historique de l'arc carpatique, pour favoriser une homogénéisation spatiale accélérée des structures socio-économiques. Ce phénomène de territorialisation paradoxale de l'espace géographique a facilité dès la chute de Ceaucescu un certain rejeu des lignes de forces d'un passé bien antérieur. On peut y voir une expression de la spatialité propre à une aire de l'entre-deux, telle que les séquences des systèmes de façonnement sont trop brèves pour produire une accumulation réelle et qu'aux moments de crise de changement de séquence, des marquages spatiaux anciens sont exhumés et retrouvent une fonctionnalité que l'on croyait dépassée. Une telle conclusion débouche sur une nécessaire exploration parallèle du phénomène de transition et de bifurcation dans les pays voisins.

* Les traitements statistiques et cartographiques ont été réalisés par Karine Emsellem. Les cartes ont été redessinées ou scannées par le Gip Reclus.

\section{Références}

BRLNET R. (1990). Chap. «Êtres géographiques», p. 164-178 de "Mondes nouveaux», t. 1 de Géographie Universelle. Paris/Montpellier: Belin/Reclus.

Espace géographique (1993). Débat «Géographie universelle et géographie dite régionale», $n^{\circ} 3$, p. 255-282.

Geografia României (1992). Bucarest: Édition de l'Académie roumaine, $4 \mathrm{vol}$.

GiLBERT A. (1988). "The new regional geography in english and french speaking countries». Progress in Human Geography, 12, p. 208-228.

GOR'JELAK G., JaLOWIECKı B. dir., (1993). «Regional question in Europe». Varsovie: Regional and local studies, $n^{\circ} 10$.

HiRSChHAUSEN B. von, (1994). «Les campagnes roumaines: la force des lieux", in Nouvelles campagnes d'Europe centrale et balkaniques. Paris: CNRS, coll. «Mémoires et Documents».

Mihallescu V. dir. (1993). En Quête d'identités. Bruxelles.

Murphy A.B. (1991). "Regions as social constructs: the gap between theory and practices». Progress in Human Geography, 15/1, p. 22-35.

REY V. (1973). "Les structures de l'espace roumain, typologie par matrice graphique ordonnable». L'Espace géographique, $\mathrm{n}^{\circ} 1$, p. 37-49.

REY V. (1975). La Roumanie, essai d'analyse régionale. Paris: SEDES, $250 \mathrm{p}$.

REY V. (1992). «L'Europe de l'Est, espace d'entre-deux». Encyclopédie de Géographie. Paris: Economica, p. 827 838.

\section{Collection Espaces modes d'emploi, $\mathbf{N}^{\circ} 1$ L'Espace géographique in the Eighties, a Transition in French Geography}

Espace géographique has been in existence for twenty years. To celebrate this the editorial board has selected 18 articles representative of French geographical research in the 1980s. The texts have been translated into English and we hope that the quality of these articles will result in an increase in the number of readers of $E G$ - whose every issue offers with each article an abstract and key-words in English.

The articles have been classified under three main headings:

Land use and development: $C$. Taillard, G. Sautter, A. Frémont, H. Beguin.

Geographical theory: M.C. Robic, F. Durand-Dastès, P. Claval, J.P. Garnier, R. Brunet, J.-B. Racine and A. Bailly, C. Raffestin and M. Bresso, Ch.P. Péguy.

The pratice of geography: D. Pumain and T. Saintjulien, Y. Guermond and J.P. Massias, M. Chevalier, A. Berque, J. Bonnemaison, R. Brunet

1993, $200 \times 260 \mathrm{~mm}, 228 \mathrm{p}$.

ISBN 2-86912-048-4 (FF 120) 